\title{
Real-time Remote Sensing Imagery Streaming over Peer-to-Peer Network
}

\author{
Huiyu Xia, Lingkui Meng \\ School of Remote Sensing and Information Engineering \\ Wuhan University \\ Wuhan, China \\ xiahuiyu@whu.edu.cn, lkmeng@whu.edu.cn
}

\author{
Feiran Xu \\ School of Resources and Environmental Engineering \\ Anhui University \\ Anhui, China \\ ahuxfr@gmail.com
}

\begin{abstract}
Motivated by the widespread use of real-time video streaming techniques over peer-to-peer networks, we propose a design of a peer-to-peer solution for real-time remote sensing imagery browsing. In this paper we firstly identify the characteristics of image browsing and mapping them to P2P-based streaming. Our design makes use of Tracker in locating remote sensing data resources. Further, a dynamic image tiles downloading queue technique which optimize image transmission is adopted. Considering that the conventional data piece selection algorithm of P2P systems has many limitations in supporting image streaming, our new approach gives each image tile a priority level before being transmitted. We use a parameter $K$ to strike the balance between high priority tiles and low priority tiles. Experimental results show that the prototype system performances much better than the traditional $\mathrm{C} / \mathrm{S}$ mode when an appropriate parameter $K$ is chosen.
\end{abstract}

Index Terms - peer-to-peer, streaming, remote sensing.

\section{INTRODUCTION}

The transmission of remote sensing image data is the basis for the application of digital earth. With the rapid growth of the remote sensing data, the traditional static way of data transmission is also replaced by the dynamic and interactive mode. Currently, Google Earth, World Wind and other mainstream digital earth platform using streaming technology, which enabling consumers to get their data continuously in real time, to transmit image data efficiently. On the other hand, these digital earth platforms, which adopt centralized architecture, need expand the scale of cluster server to face the swift growth of remote sensing data size and user scale and then costs large amounts of investment as well as energy. Even more, it brings several environmental problems ${ }^{[1]}$. The traits of P2P technology such as decentralized server and scalable architecture provide a feasible way to solve the bottleneck problem of centralized architecture.

After ten years of development, P2P technology has been successfully applied in fields of file sharing, media streaming, spatial image data transmission and so on ${ }^{[2,3]}$. In this paper we present the mechanism of remote sensing image streaming in P2P environment and design an optimized piece selection algorithm of image streaming. We also build a simulation system to test the efficiency of the algorithm.

\section{RELATED WORK}

Recently, spatial image delivery based on P2P has been attracted by many scholars. Yi Liu et al.[4] proposed a design for efficiently spatial data delivery over P2P network, which adopted grid-based peer group for resource locating and peer selection mechanisms to optimize the results of resource locating. Zhan$\mathrm{Wu} \mathrm{Yu}$ et al.[5] proposed a new model for terrain data transmission and a structure for client node. In their model they used rarest first block selection strategy and optimized peer selection algorithm based on performance and bandwidth available. Wei Wang[6] designed a 3D scenes roaming system in $\mathrm{P} 2 \mathrm{P}$ environment, which adopted a progressive scenes replacement and neighbor nodes selection mechanism. Our work focuses on image data streaming in P2P network, benefiting from experiences of real-time video streaming, in consideration of the real network condition including flash crowd.

\section{Remote Sensing ImAge StREAming}

Image streaming is an efficient technique which enabling image data to display and download simultaneously based on partitioning image data into blocks. It means that users can browse their requested remote sensing images even when they are downloading. Image streaming significantly reduces waiting time by transmitting image blocks in region of interest to users without waiting for all downloading tasks are completed. In the image streaming, the basic transmission unit is known as image tiles. Tile is the smallest unit of image blocks, and it has a unique index code globally. Clients request different scope of multiresolution remote sensing images for browsing and other operations. Spatial data engine calculates the mathematical relationships between the latitude and longitude of user's region of interest and the spatial index of tiles, then returns the image tiles collection within user's browsing region. Thus, with the updates of user's view point, different image tiles will be continuously transmitted to the client in real-time. In this type of image streaming, large amounts of data requests will be produced by user's frequency behavior such as Pan and Zoom in browsing images. However, these requests are not totally useful for user. Figure 1 shows the usefulness of the requests created by pan operation. 


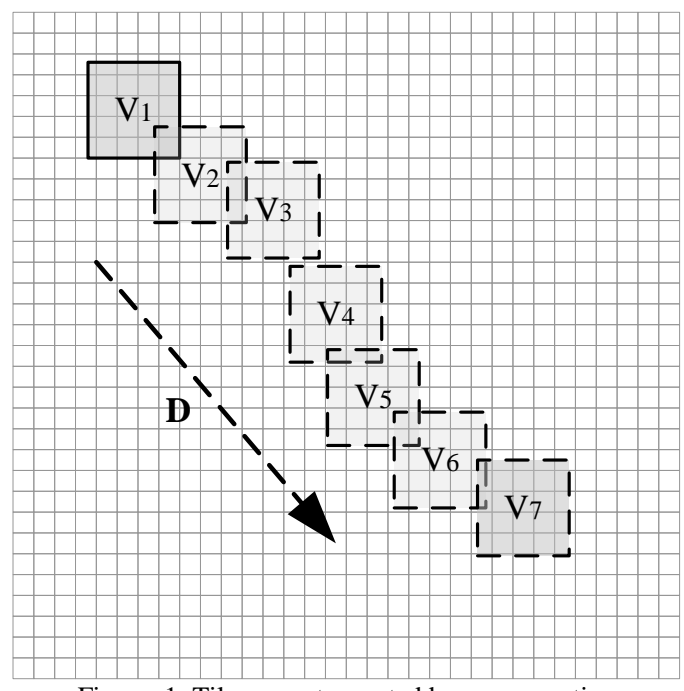

Figure. 1 Tile requests created by pan operation

In Figure 1, the background grids refer to the image data which have been sliced and indexed. Every grid cell represents an image tile. $\mathrm{V}_{i}(i=1 \sim 7)$ refer to the rectangular regions generated by user's Pan operation in direction D, image tiles collections covered by these regions are the data that should be finally requested. For users, $\mathrm{V}_{7}$ is the target region of pan operation, and $\mathrm{V}_{2} \sim \mathrm{V}_{6}$ are the temporary regions which are generated during pan operation (assuming that $\mathrm{V}_{1}$ is the last target region of interest). So the tiles included in $\mathrm{V}_{2} \sim \mathrm{V}_{6}$ have a lower request priority (LowPriority), and tiles included in $\mathrm{V}_{7}$ have a higher request priority (HighPriority). Defining image tiles sets corresponding to $\mathrm{V}_{i}$ as $\mathrm{P}\left[\mathrm{V}_{i}\right]$, then $\mathrm{P}\left[\mathrm{V}_{7}\right] \in$ HighPriority, $\mathrm{P}\left[\mathrm{V}_{2}\right] \sim \mathrm{P}\left[\mathrm{V}_{6}\right] \in$ LowPriority.

Priority of image tiles plays an important role in image streaming. As the HighPriority image tiles are within the user's current view region, they should be downloaded before the LowPriority tiles which are out of user's view region in order to make sure users can get their information in time.

\section{CONTENT LOCATING MECHANISM}

Content locating is an important realization mechanism in remote sensing image streaming based on P2P. Our work adopts the traditional way to locate resources based on tracker, which registers all nodes in $\mathrm{P} 2 \mathrm{P}$ network and responds each peer to return a list of neighbor peers selected randomly. Several researches on spatial data transmission in $\mathrm{P} 2 \mathrm{P}$ environment have adopted grouping strategy to locate resources ${ }^{[7]}$. Grouped P2P network is commonly used in unstructured P2P network to locate contents. It groups nodes according to their interesting contents or resources which have been downloaded in order to manage the relationship between each node and resources ${ }^{[5,6]}$. However, grouping strategy is inefficient in spatial resources locating. As the image requests are generated by the varying of user's region of browsing, they are destined to have randomness because of the differences of users' demands. The cached image data of each node cannot have any geographic positional correlation even they are in the same geographical area. Nodes can't be grouped according to user's location because there is no definite relationship between user attributes and their requests. Moreover, setting grouped unit as a certain range of image tiles will result in excessively high maintenance costs and bring many limitations.

Tracker records and manages global nodes information. When a newly joined node submits a request, tracker responds it a list of neighbor peers, selected randomly in global nodes. The new node will connect to each peer respectively after receiving this list. Node exchanges have message continuously with its connected neighbor peers to tell each other they have downloaded certain new image tile. So each node can get the distribution of resources in its connected network, and then requests data when needed. These random graphs have very good robustness properties, and it's suitable for large-scale network applications ${ }^{[7]}$.

\section{PEER DOWNLOADING STRATEGIES}

When new node joining the network, it will submit an initial message to tracker. Tracker authorizes the node and then responds it with a list of neighbor peers. The newly joined node connects these peers for data exchanging and begins its life cycle after receiving the response. In traditional $\mathrm{P} 2 \mathrm{P}$ file sharing system, the download tasks of a peer are single files. So storage space is previously established according to the files size before receiving data. However, there is no "single file" concept in remote sensing image streaming since the data size is too large. Therefore, the cache capacity in disk should be defined before the initialization of image streaming task. Peers then use the resources cached in the disk to exchange with other peers. In our image streaming we used a dynamic task queue to download image tiles. As shown in Figure 2, ViewRequestQueue represents the view regions request queue generated by user's browsing. TileQueue represents the image tiles queue corresponding to various regions. Define the tiles in TileQueue as HighPriority and LowPriority according to previous definition. As the user changes the current view regions, new tile request $\mathrm{P}\left[\mathrm{V}_{3}\right]$ will add into the head of TileQueue and the priority of the tiles in TileQueue will shift accordingly.

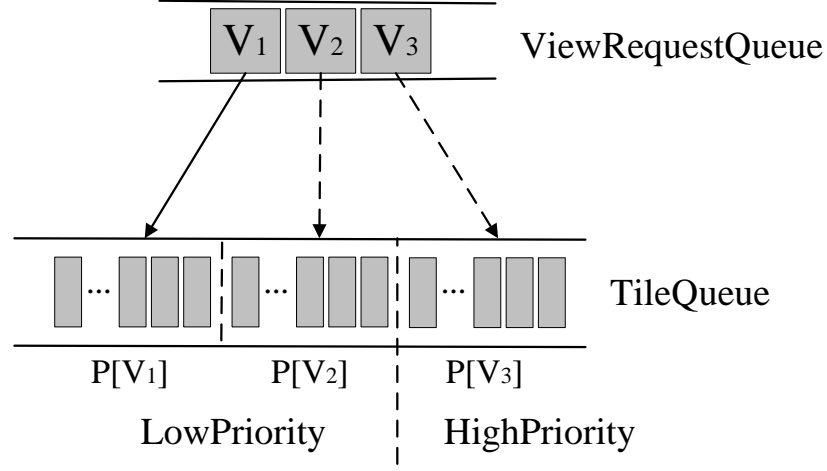

Figure. 2 Dynamic downloading queue in BTI-Peer

TileQueue is the key to access to image streaming in peers. It represents the most desired image tiles of the peer node currently. Peer monitors the changes of TileQueue and updates data block 
of interest dynamically. However, because of the high variability of the download queue, we need to constraint the TileQueue in multiple terms. First, because of the possibility that the view region may be overlapped, the queue may have many reduplicate tiles. The peer should detects these reduplicate tiles and remove them when refreshing the TileQueue. Secondly, with the queue length increasing, the tiles have the lower timeliness if they are closer to the tail of the download queue. Therefore, we constrain the queue length as $\mathrm{N}$. If the length of queue, let's say $m$, is bigger than $N$, then remove $(m-N)$ blocks from the bottom of TileQueue.

\section{IMAGE TILE SELECTION ALGORITHM}

Traditional P2P file sharing system uses local rarest first (LRF) strategy to select data blocks during downloading. The LRF algorithm does a good job of making sure that each peer has the pieces that other peers desired, so uploading can be done when wanted. It also makes sure that pieces which are more common are left for later, so the likelihood that a peer which currently is offering upload will later not have anything of interest is reduced. However, this approach could not meet the need of service quality in image streaming. If we using LRF strategy to select piece, then the high priority image tiles cannot arrive in time, user's waiting time will increase and the QoS is destroyed. Therefore, in order to take care of QoS and the efficient of P2P system, we designed a new image tile selection algorithm.

When peer A obtaining image tiles from peer B, it firstly mapping the priorities of the available data set in peer B, according to the priorities that have been identified in TileQueue. As shown in Figure 3, ATS (Available Tiles Set) represents the available image tile set from other peers. We define the tiles priority in ATS as HighPriority and LowPriority. Set a probability value $K$ from $(0,1]$. When peer A downloading data from peer B, it chooses with the probability $K$ to download a tile in HighPriority tiles using LRF strategy and with probability $1-K$ to download a tile contained in LowPriority tiles using LRF. It is worth noting that the tiles usually have the same rareness. But as we have limited the set in LRF selection, our algorithm will select one tile from these tiles randomly.

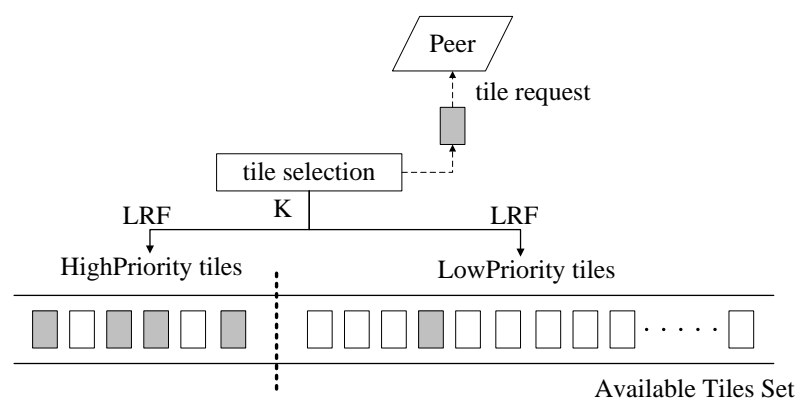

Figure. 3 Piece selection algorithm of image tiles

The key of this algorithm is the choice of probability value $K$. When value $K$ getting larger, the images in user's current region of interest will arrive earlier. On the other hand, due to the global rareness of image tiles, the downloading peer cannot get data blocks that other peers interests. So it will be blocked by other peers usually and result in low transfer rate.

\section{EXPERIMENTAL EVALUATION}

\section{A. Simulation Environment Settings}

In order to evaluation the effectiveness of the mechanism and algorithm in our work, we developed a simulation system. In this simulator we have included all the functionalities of the traditional P2P system and we have also incorporated the dynamic downloading queue and the image tile selection algorithm in peer. The remote sensing images are partitioned and indexed using quad-tree structure. Each tile in system has a unique index code. Peer generates image requests continually in its life cycle. Assuming that the region of interest size is fixed, setting one region includes $M$ image tiles. Initialized system includes tracker and seed nodes which have all the image resource. Set upload/download bandwidth after peers joining the system, and then give them initialized image tiles. Peer's upload/download bandwidth keeps stable in its life cycle. Moreover, the simulation system records all the examine indicators using system $\log$.

\section{B. Experiments and Results Analysis}

The image transmission efficiency and the service quality are two important aspects in testing the performance of the algorithm. Time spent in the image tiles transmission can be used to measure the transmission efficiency of image streaming between nodes. For the quality of image service, we adopt the proportion of tiles in user's region of interest within all the arriving tiles as the main indicator. We present TAI (Tiles Arrival Index) to comprehensively reflect these two important indicators. TAI can be expressed as following:

$$
\mathrm{TAI}=L /\left(t-S D_{\text {peer }}\right)
$$

In formula (1), $L$ refers to the number of tiles included in the region of interest. $t$ represents the transmission time of $M$ blocks. $S D_{\text {peer }}$ is the time for peer initializing. As $L$ getting larger, the number of image tiles included in region of interest is increasing, so users can get their information in time. Low value $\left(t-S D_{\text {peer }}\right)$ indicates that the transmission is on a high speed. To sum up, the higher value of TAI represents that the algorithm has the better performance.

1) chosen of $K$

Value $K$ is the key to tile selection algorithm. The experiment firstly tests the relationship between $K$ and TAI. Assuming that peers join the simulation system according to Poisson process, the test records TAI value of each node in transferring 20 tiles and uses means of 50 tests. 


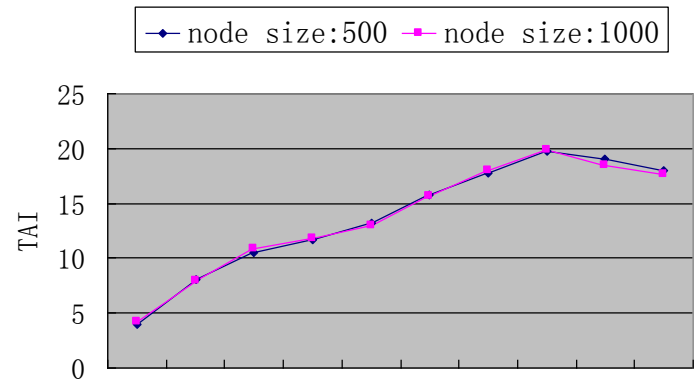

$\begin{array}{lllllllllll}0.1 & 0.2 & 0.3 & 0.4 & 0.5 & 0.6 & 0.7 & 0.8 & 0.9 & 1.0\end{array}$

Figure. 4 Variation of TAI under different parameter $\mathrm{K}$

Figure 4 shows the variation of TAI under different value $\mathrm{K}$ when the node sizes are 500 and 1000 respectively. When node size is 500, the TAI value is getting larger with the increasing of $K$; When $K$ equal to 0.8 , TAI reaches its peak value; TAI begins to decrease when $K$ is bigger than 0.8. We can also find that when node size reaches 1000 , the variation of TAI is almost the same.

The results can be explained by the principle of the tile selection algorithm. The lower $K$ value means that the reaching rate of tiles included in region of interest is lower. In this condition although the algorithm has considered the rareness of LowPriority tiles, the quality of image service is destroyed because reached tiles are not included in the current user's region, and TAI is in a low level. But tiles in user's region increases when $K$ increasing, so TAI is also getting larger. The system performance is optimum when $K$ increasing to 0.8. After that, because of the frequency of LRF selection in HighPriority tiles is too high, the global rareness principle is undermining, TAI value begins to decrease as the transmission speed falling.

\section{2) Data Server Load}

The amount of average image tile requests accepted can be a good indicator to measure the data server load. The experiment compared the data server workload between $\mathrm{C} / \mathrm{S}$ mode and $\mathrm{P} 2 \mathrm{P}$ mode. Figure 5 shows that with the increasing number of nodes, the server workload rapidly increased in the traditional $\mathrm{C} / \mathrm{S}$ mode. However, the workload grows slowly in P2P mode. When the node size reaches 500, the server load in P2P mode is only 15 percent of server load in $\mathrm{C} / \mathrm{S}$ mode, so it greatly reducing the server workload.

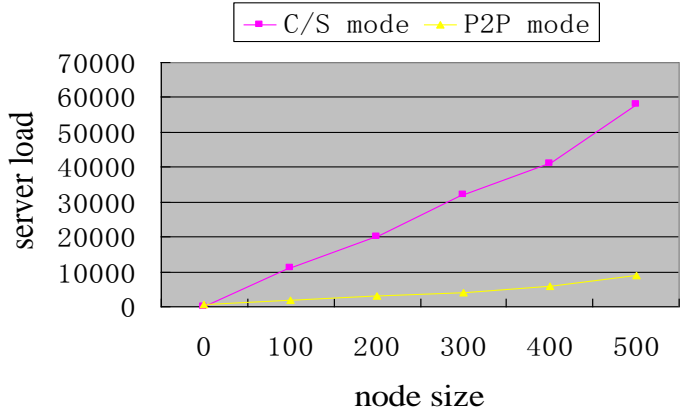

Figure. 5 Comparison of server load between $\mathrm{P} 2 \mathrm{P}$ and $\mathrm{C} / \mathrm{S}$ modes
Figure 6 shows the comparison of server load between C/S mode and P2P mode when 500 nodes join the system in the same time (flash crowd scenario). With the system runtime increasing, server load stays at a high state in C/S mode. However the load curve has an obvious downward trend in $\mathrm{P} 2 \mathrm{P}$ mode, and being stabilized after the system running to $80 \mathrm{~min}$. The server load is only about 14 percent of workload in $\mathrm{C} / \mathrm{S}$ mode under the same condition.

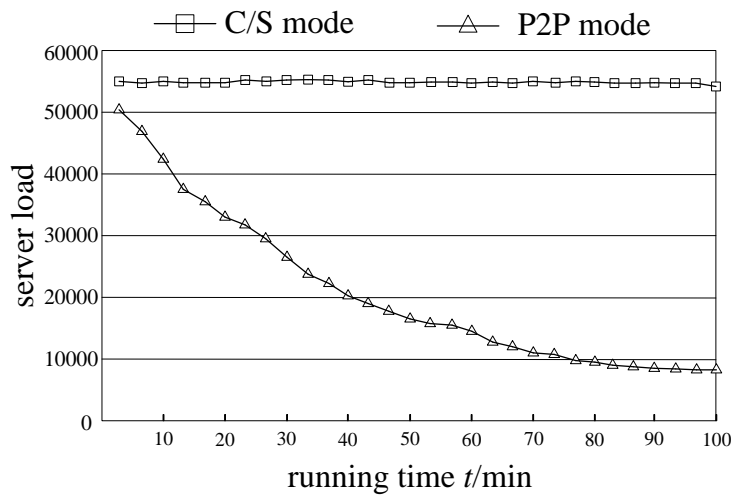

Figure. 6 Comparison of server load in the scenario of flash crowd

\section{CONCLUSION}

This paper proposes a mechanism for remote sensing image streaming in $\mathrm{P} 2 \mathrm{P}$ environment, and describes the peer downloading strategies and an image tile selection algorithm. We also build a simulation system to verify the efficiency of our proposed algorithm. The experimental results show that the tile selection algorithm achieves the best performance when the parameter $K$ equals to 0.8 . In addition, our approach reduces the data server workloads significantly compared to the traditional $\mathrm{C} / \mathrm{S}$ architecture.

\section{REFERENCES}

[1] New Greenpeace Study Reveals Dirty Energy Behind Internet's Growth[EB/OL].[2011-04-21].

http://www.greenpeace.org/international/en/press/releases/NewGreenpeace-Study-Reveals-Dirty-Energy-Behind-InternetsGrowth--/.

[2] L. Yi, G. Jianya, WU Huayi. P2P Based Efficient On-line Spatial Images Delivery[C]//Proceedings of Geoinformatics'2007. Nanjing:SPIE, 2007.

[3] A. Vlavianos, M. Iliofotou, M. Faloutsos. BiToS: Enhancing BitTorrent for Supporting Streaming Applications[C]//9th IEEE Global Internet Symposium. Barcelona, Spain, 2006.

[4] L. Dan, X. Wenjun. Spatial Region Query Based on Grouping P2P Network[J]. Computer Engineering, 2009,35(17), pp. 49-51.

[5] V. Sahota, et al. A Grouped P2P Network for Scalable Grid Information Services[J]. Peer-to-Peer Networking and Applications, 2009, 2.pp. 3-12.

[6] K. Sripanidkulchai, B. MAGGS, et al. Efficient Content Location Using Interest-based Locality in Peer-to-Peer Systems[C]//Proceedings of IEEE INFOCOM, Mar. 2003.

[7] B. Cohen. Incentives Build Robustness in BitTorrent $[\mathrm{C}] / / 1 \mathrm{st}$ Workshop on the Economics of Peer-2- Peer Systems, Berkley, CA, June 5-6 2003. 